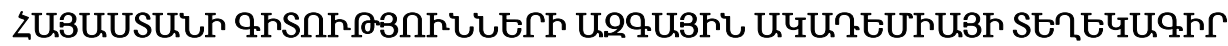 ИЗВЕСТИЯ НАЦИОНАЛЬНОЙ АКАДЕМИИ НАУК АРМЕНИИ
}

\author{
Uthuminlqu \\ 73, №2, 2020 \\ УДК 517.934 \\ Doi- http://doi.org/10.33018/73.2.6

\section{HYBRID CONTROL OF A MOTION OF AN UNMANNED AERIAL VEHICLE, CARRYING AN INVERTED PENDULUM Shahinyan A. S.}

Механика

Keywords: Dynamical systems, hybrid control, optimal stabilization, quadcopter, inverted pendulum, phase trajectories, virtual modeling.

\author{
Шагинян А. С.
}

Гибридное управление движением беспилотного летательного аппарата, несущего перевернутый маятник

Ключевые слова: динамические системы, гибридное управление, оптимальная стабилизация, квадрокоптер перевернутый маятник, фазовые траектории, виртуальное моделирование.

Рассматривается проблема управления движением беспилотного летательного аппарата (БПЛА), несущего перевернутый маятник. Описана взаимосвязанная динамика беспилотного летательного аппарата и перевернутого маятника. Для решения, линеаризованной на основе виртуального моделирования системы математической задачи управления, применен новый: гибридный метод управления системой. Решена линеаризованная задача управления системой.

Управляющие воздействия на систему, а также фазовые траектории движения составляющих системы приведены в виде графиков функций.

Tuhhijuui U. U.

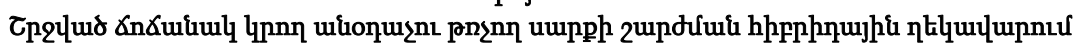

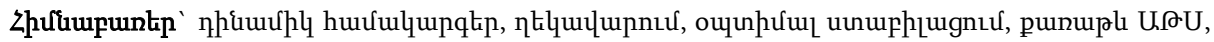

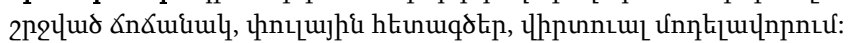

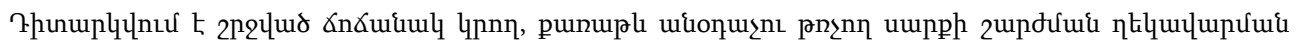

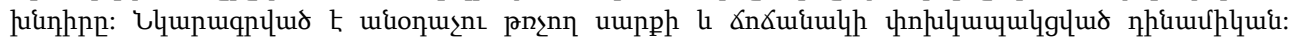

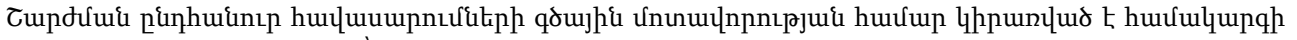

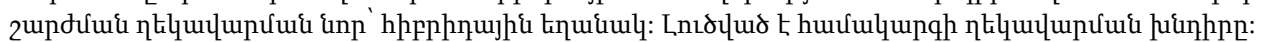

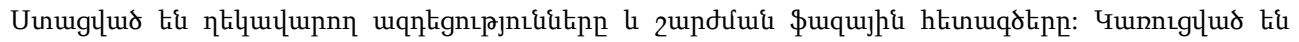
пnuigg qnuфhlqutin:

The control problem of the movement of an unmanned aerial vehicle (UAV) carrying an inverted pendulum is considered. The interconnected dynamics of an unmanned aerial vehicle and an inverted pendulum are described. To solve the control mathematical linearized problem on the basis of virtual modeling of the system, a new one was applied: a hybrid method of the system control.

The linearized system control problem is solved. The control actions on the system, as well as phase trajectories of the components of the system are given in the form of graphs of functions.

\section{Introduction}

Unmanned Aerial Vehicles (UAVs) are, in general, flying vehicles which do not require a person onboard to be controlled. UAVs can be also considered as flying robots. The history of UAVs starts from 1880s when those robots were used mainly in military purposes. One of the first ways people used UAVs was as high-altitude photographers. However, these extremely simple flying drones were not popular until the times of first world war. Several years after drones acquired popularity they were applied in military missions like 
elimination of unexploded bombs, assessment damaged buildings in an area, exploration of enemy forces, etc. [1].

Decades later when science and technology developed side by side UAVs also became more advanced and they are capable of completing much more complex missions not only in military forces but also for civilians. Nowadays applications of UAVs include missions like mapping, pollution and land monitoring, powerline inspection, fire detection, agriculture, and among other applications.

Among all UAVs, the quadrotors of most interest amongst scientists and researchers because of their structure simplicity, cheap price and extremely huge dynamic potential. A decent amount of papers are dedicated to dynamics and control of these kind of robots namely papers present control techniques like Proportional Differential (PD) control [2,3], Proportional Integral Differential (PID) control [4, 5], control of position and orientation by vision [6], sliding mode control [1, 7], fuzzy logic [8, 9], and adaptive control in [10].

In [12] a UAV is considered with an inverted pendulum mounted on its body. The paper makes trajectory constraints on the UAV-Pendulum system and, hence derives a Linear Quadratic Regulator (LQR) controller to stabilize the system along the trajectory or the hover point. First, they make the system hover in a point and stabilize around that point and then they want the UAV to move around a point center in a circular trajectory

In this paper dynamics of a UAV is considered alongside with an inverted pendulum mounted on the UAV. The dynamics of the pendulum is presented with respect to the UAV and then both models are combined into one using some rules and theorems of the Theoretical Mechanics. After linearizing the model, a novel hybrid method of control is applied to the system to solve the control problem.

The hybrid model we applied is as follows. We first stabilize optimally the pendulum using the motion of the UAV as control inputs and then we use the optimal stabilizing control inputs to drive the UAV-Pendulum system to a desired position.

The results we gained i.e. the control inputs and state trajectories are shown in form of graphs which were generated from virtual simulations.

\section{Modelling of the System}

To derive the pure theoretical dynamics of a UAV let us fix a coordinate system Oxyz . Let

$O$ be the origin. We will also need another coordinate system $O_{B} x_{B} y_{B} z_{B}$ fixed in the center of mass $O_{B}$ of the UAV (fig. 1). The torques and forces generated by each of the propellers are shown in the Figure 1. The propellers are numbered 1 to 4 [13].
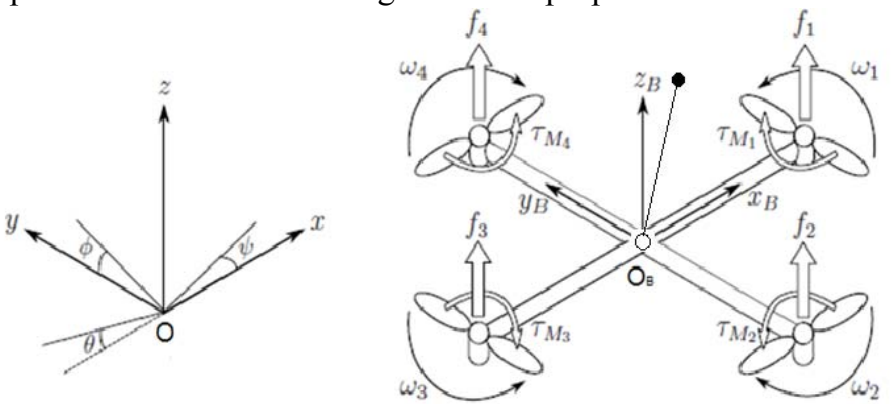

Figure 1.
Let $\xi=\left(\begin{array}{lll}x & y & z\end{array}\right)^{T}$ be the coordinates of the center of mass of the UAV with respect to the system Oxyz . As mentioned above, the center of the mass of the UAV coincides with the origin of the coordinate system $O_{B} x_{B} y_{B} z_{B}$. Let us

describe the inclined position of the UAV about the point $O_{B}$ using yaw, pitch and roll 
angles. Let $\Phi$ be the pitch angle, $\Theta$ be the roll angle and, finally, let $\Psi$ be the yaw angle. Then we will have two vectors describing the position of the UAV. Those are the following:

$$
\xi=\left(\begin{array}{lll}
x & y & z
\end{array}\right)^{T}, \eta=\left(\begin{array}{lll}
\Phi & \Theta & \Psi
\end{array}\right)^{T}
$$

In the coordinate system the linear velocities $\bar{V}_{B}$ and the angular velocities $\bar{v}$ are the following

$\bar{V}_{B}=\left(\begin{array}{lll}V_{B x} & V_{B y} & V_{B z}\end{array}\right)^{T}, \quad \bar{v}=\left(\begin{array}{lll}p & q & r\end{array}\right)^{T}$

In this setup we will have the dynamics of the system as given below [13;15].

$$
\left\{\begin{array}{l}
\ddot{x}=\frac{T}{m} c_{\Psi} s_{\Theta} c_{\Phi}+\frac{T}{m} s_{\Psi} s_{\Phi} ; \quad \ddot{y}=\frac{T}{m} s_{\Psi} s_{\Theta} c_{\Phi}-\frac{T}{m} c_{\Psi} s_{\Phi} ; \quad \ddot{z}=-g+\frac{T}{M} c_{\Theta} c_{\Phi} ; \\
\dot{\Phi}=p+\frac{s_{\Phi} s_{\Theta}}{c_{\Theta}} q+\frac{c_{\Phi} s_{\Theta}}{c_{\Theta}} r ; \quad \dot{\Theta}=c_{\Phi} q-s_{\Phi} r ; \quad \dot{\Psi}=\frac{s_{\Phi}}{c_{\Theta}} q+\frac{c_{\Phi}}{c_{\Theta}} r ; \\
\dot{p}=\frac{\left(I_{y y}-I_{z z}\right) q r}{I_{x x}}-I_{r} \frac{q}{I_{x x}} \omega_{\Gamma}+\frac{\tau_{\Phi}}{I_{x x}} ; \quad \dot{q}=\frac{\left(I_{z z}-I_{x x}\right) p r}{I_{y y}}-I_{r} \frac{p}{I_{y y}} \omega_{\Gamma}+\frac{\tau_{\Theta}}{I_{y y}} ; \\
\dot{r}=\frac{\left(I_{x x}-I_{y y}\right) p q}{I_{z z}}-I_{r} \frac{q}{I_{z z}} \omega_{\Gamma}+\frac{\tau_{\Psi}}{I_{z z}} ;
\end{array}\right.
$$

Where the following notations are used:

$C_{\alpha}:=\cos \alpha, S_{\alpha}:=\sin \alpha, M=m_{U A V}+m_{P}$

$\tau_{B}=\left(\begin{array}{c}\tau_{\Phi} \\ \tau_{\Theta} \\ \tau_{\Psi}\end{array}\right)=\left(\begin{array}{c}l k\left(-\omega_{2}^{2}+\omega_{4}^{2}\right) \\ l k\left(-\omega_{1}^{2}+\omega_{3}^{2}\right) \\ \sum_{i} \tau_{i}\end{array}\right)$

$T=\sum_{i} F_{i}=\sum_{i} k \omega_{i}^{2}, \quad \vec{T}=\left(\begin{array}{lll}0 & 0 & T\end{array}\right)^{T}$

As for the mathematical model of the pendulum we will consider its dynamics in the coordinate system $O_{B} X_{B} y_{B} Z_{B}$.

So, the dynamics of the pendulum will be as shown below. [4]

$$
\left\{\begin{array}{c}
\ddot{x}_{p}=\frac{1}{\left(L^{2}-y_{p}^{2}\right) \zeta^{2}} \cdot\left(\begin{array}{l}
-x_{p}^{4} \ddot{x}-\left(L^{2}-y_{p}^{2}\right) \ddot{x}-2 x_{p}^{2}\left(y_{p} \dot{x}_{p} \dot{y}_{p}-\left(L^{2}-y_{p}^{2}\right) \ddot{x}\right) \\
+x_{p}^{3}\left(\dot{y}_{p}^{2}+y_{p} \ddot{y}_{p}-\zeta(g+\ddot{z})\right)
\end{array}\right. \\
\left.+x_{p}\left(-L^{2} y_{p} \ddot{y}_{p}+y_{p}^{3} \ddot{y}_{p}+y_{p}^{2}\left(\dot{x}_{p}^{2}-\zeta(g+\ddot{z})\right)+L^{2}\left(-\dot{x}_{p}^{2}-\dot{y}_{p}^{2}+\zeta(g+\ddot{z})\right)\right)\right) \\
\ddot{y}_{p}=\frac{1}{\left(L^{2}-x_{p}^{2}\right) \zeta^{2}} \cdot\left(\begin{array}{l}
-y_{p}^{4} \ddot{y}-\left(L^{2}-x_{p}^{2}\right) \ddot{y}-2 y_{p}^{2}\left(x_{p} \dot{x}_{p} \dot{y}_{p}-\left(L^{2}-x_{p}^{2}\right) \ddot{y}\right) \\
+y_{p}^{3}\left(\dot{x}_{p}^{2}+x_{p} \ddot{x}_{p}-\zeta(g+\ddot{z})\right)
\end{array}\right. \\
\left.+y_{p}\left(-L^{2} x_{p} \ddot{x}_{p}+x_{p}^{3} \ddot{x}_{p}+x_{p}^{2}\left(\dot{y}_{p}^{2}-\zeta(g+\ddot{z})\right)+L^{2}\left(-\dot{x}_{p}^{2}-\dot{y}_{p}^{2}+\zeta(g+\ddot{z})\right)\right)\right)
\end{array}\right.
$$

Using the formula of center of mass of a system 
$\bar{X}_{C}=\frac{m_{1} \bar{r}_{1}+m_{2} \bar{r}_{2}}{m_{1}+m_{2}}$

where $\bar{r}_{1}=\bar{\xi}=\left(\begin{array}{lll}x & y & z\end{array}\right)^{T}$ and $\bar{r}_{2}=\bar{r}_{p}=\left(\begin{array}{lll}x_{p} & y_{p} & z_{p}\end{array}\right)^{T}$, we can find the coordinates of center of mass of our UAV-Pendulum system in the coordinate system Oxyz. Let $m_{1}=m_{2}=1$, then we will have

$\left\{x_{c}=x+\frac{1}{2} x_{p} ; y_{c}=y+\frac{1}{2} y_{p} ; z_{c}=z+x+\frac{1}{2} \sqrt{l_{p}^{2}+x_{p}^{2}+y_{p}^{2}}\right.$

To get the state space model of the UAV-Pendulum system we introduce the notations as shown below

$$
\left\{\begin{array}{llll}
x_{1}=x_{c} ; & x_{2}=\dot{x}_{c} ; & x_{3}=y_{c} ; & x_{4}=\dot{y}_{c} ; \\
x_{5}=z_{c} ; & x_{6}=\dot{z}_{c} ; & x_{7}=\Phi ; & x_{8}=\Theta ; \\
x_{9}=\Psi ; & x_{10}=p ; & x_{11}=q ; & x_{12}=r ; \\
x_{13}=x_{p} ; & x_{14}=\dot{x}_{p} ; & x_{15}=y_{p} ; & x_{16}=\dot{y}_{p} ;
\end{array}\right.
$$

We linearize the dynamics around the origin of the fixed coordinate system. So, we finally get.

$$
\left\{\begin{array}{llll}
\dot{x}_{1}=x_{2} ; & \dot{x}_{2}=\frac{g}{2 l_{p}} x_{13} ; & \dot{x}_{3}=x_{4} ; & \dot{x}_{4}=\frac{g}{2 l_{p}} x_{15} ; \\
\dot{x}_{5}=x_{6} ; & \dot{x}_{6}=u_{1} ; & \dot{x}_{7}=x_{10} ; & \dot{x}_{8}=x_{11} ; \\
\dot{x}_{9}=x_{12} ; & \dot{x}_{10}=\frac{u_{2}}{I_{x x}}-\frac{g}{I_{x x}} x_{15} ; & \dot{x}_{11}=\frac{u_{3}}{I_{y y}}-\frac{g}{I_{y y}} x_{13} ; & \dot{x}_{12}=\frac{u_{4}}{I_{z z}} ; \\
x_{13}=x_{14} ; & x_{14}=-g x_{8}+\frac{g}{l_{p}} x_{13} ; & x_{15}=x_{16} ; & x_{16}=g x_{7}+\frac{g}{l_{p}} x_{15} .
\end{array}\right.
$$

where $u_{1}=\frac{T}{M}-g, u_{2}=\tau_{\Phi}, u_{3}=\tau_{\Theta}, u_{4}=\tau_{\Psi}$.

Using Kalman's rule one can check that the system (7) is fully controllable. So, now we are in a point where we can define the problem and we can go ahead to show the way we solved it.

\section{Problem Definition:}

Given the system (7), the initial position of the system $x_{i}(0)=x_{i, 0}, i=\overline{1,16}$ and the final position $x_{i}\left(t_{1}\right)=x_{i, 1}, i=\overline{1,16}$, find control inputs $u_{i}, i=\overline{1,4}$ such that it drives the system from the given initial position to the given final.

As one can notice this control problem is not an optimal control problem.

\section{Problem Solution.}

Our approach to the problem solution was the following. First, we ensure that the pendulum remains where it should be. We do this by applying optimal control input stabilizers inside the coordinate system $O_{B} x_{B} y_{B} z_{B}$. And after we know that the pendulum will remain inverted (will not drop) we proceed to the control problem. Let us now define a subproblem of optimal stabilization for the subsystem 
$\left\{x_{13}=x_{14} ; x_{14}=-g u_{5}+\frac{g}{l_{p}} x_{13} ; x_{15}=x_{16} ; x_{16}=g u_{6}+\frac{g}{l_{p}} x_{15}\right.$

Note that here we use the notation

$\left\{x_{8}=u_{5} ; x_{7}=u_{6}\right.$

Now the subproblem will be the defined as follows.

4. Problem Definition: Given the system (8), the initial position of the system $x_{i}(0)=x_{i, 0}, i=\overline{13,16}$, find control inputs $u^{0}=\left(\begin{array}{ll}u_{5}^{0} & u_{6}^{0}\end{array}\right)^{T}$ such that it drives the system from the given initial position to asymptotically stable position while minimizing the linear quadratic regulator

$J[\bullet]=\int_{0}^{\infty}\left(x_{14}^{2}+x_{16}^{2}+u_{5}^{2}+u_{6}^{2}\right) d \tau$

Solution: Notice that the system (8) can be divided into two subsystems which are

$\left\{x_{13}=x_{14} ; x_{14}=\frac{g}{l_{p}} x_{13}-g u_{5}\right.$
$\left\{x_{15}=x_{16} ; x_{16}=\frac{g}{l_{p}} x_{15}+g u_{6}\right.$

We will show the solution steps for one of the systems (say (8.1)) as both of them are solved absolutely identically.

We choose to solve the optimal stabilization problem by using Lyapunov-Bellman method. In general, the method says that the optimal control input $u^{0}$ has to satisfy the optimization equation as given below

$$
\min _{u}\left(\nabla V(x)(A x+B u)+\left(x^{T} Q x+u^{T} R u\right)\right)=0
$$

Where

$\mathfrak{B}[\bullet]=\nabla V(x)(A x+B u)+\left(x^{T} Q x+u^{T} R u\right)$

(11) is Bellman's expression for the linear time-invariant control systems. So, in our case for the system (8.1) we will have

$\mathfrak{B}[\bullet]=\frac{\partial V}{\partial x_{13}} x_{14}+\frac{\partial V}{\partial x_{14}}\left(\frac{g}{l_{p}} x_{13}-g u_{5}\right)+x_{14}^{2}+u_{5}^{2}$

It is obvious that the value of $u_{5}^{0}$ which optimizes (10) is the extremum of (12). Thus, we will have

$u_{5}^{0}=\frac{g}{2} \frac{\partial V}{\partial x_{14}}$

By substituting (13) back into (12) we get the following.

$\frac{\partial V}{\partial x_{13}} X_{14}+\frac{g}{l_{p}} X_{13} \frac{\partial V}{\partial x_{14}}-\frac{g^{2}}{4}\left(\frac{\partial V}{\partial x_{14}}\right)^{2}+x_{14}^{2}=0$

Here $V=V\left(x_{13}, x_{14}\right)$ is the Lyapunov function for the system (8.1) and we search for it in the form 
$V=\frac{1}{2}\left(c_{11} x_{13}^{2}+2 c_{12} x_{13} x_{14}+c_{22} x_{14}^{2}\right)$

Putting (15) into (14) we get a algebraic equation which have the form

$$
\left(c_{11} x_{13}+c_{12} x_{14}\right) x_{14}+\frac{g}{l_{p}}\left(c_{12} x_{13}+c_{22} x_{14}\right) x_{13}-\frac{g^{2}}{4}\left(c_{12} x_{13}+c_{22} x_{14}\right)^{2}+x_{14}^{2}=0
$$

From (16) the following system of equations will follow

$$
\left\{\begin{array} { l } 
{ \frac { g } { l _ { p } } c _ { 1 2 } - \frac { g ^ { 2 } } { 4 } c _ { 1 2 } ^ { 2 } = 0 } \\
{ c _ { 1 2 } - \frac { g ^ { 2 } } { 4 } c _ { 2 2 } ^ { 2 } + 1 = 0 } \\
{ c _ { 1 1 } + \frac { g } { l _ { p } } c _ { 2 2 } - \frac { g ^ { 2 } } { 2 } c _ { 1 2 } c _ { 2 2 } = 0 }
\end{array} \Rightarrow \left\{\begin{array}{l}
c_{11}=\frac{2}{l_{p}} \sqrt{\frac{g l_{p}+4}{g l_{p}}} \\
c_{12}=\frac{4}{g l_{p}} \\
c_{22}=\frac{2}{g} \sqrt{\frac{g l_{p}+4}{g l_{p}}}
\end{array}\right.\right.
$$

Here the shown solutions are the ones which make $V=V\left(x_{13}, x_{14}\right)$ positive definite. Finally, to get $u_{5}^{0}=u_{5}^{0}\left(x_{13}, x_{14}\right)$ we put (17) into (15) and put what we get into (13). That gives us

$$
u_{5}^{0}=\frac{2}{l_{p}} x_{13}+\sqrt{\frac{g l_{p}+4}{g l_{p}}} x_{14}
$$

To obtain $u_{5}^{0}=u_{5}^{0}(t)$ we simply need to substitute (18) into (8.1) and integrate the system. We will get

$$
\begin{aligned}
& u_{5}^{0}=\sqrt{\frac{g l_{p}+4}{g l_{p}}} \frac{\left(e^{\frac{t\left(-g l_{p}-g l_{p} \sqrt{\frac{g l_{p}+4}{g l_{p}}}\right)}{2 l}}-e^{\left.\frac{t\left(g l_{p}-g l_{p} \sqrt{\frac{g l_{p}+4}{g l_{p}}}\right)}{2 l_{p}}\right)}\right.}{2 l_{p}}- \\
& -\sqrt{\frac{g l_{p}+4}{g l_{p}}} \frac{\left(e^{\frac{t\left(-g l_{p}-g l_{p} \sqrt{\frac{g l_{p}+4}{g l}}\right)}{2 l_{p}}}-e^{\left.\frac{t\left(g l_{p}-g l_{p} \sqrt{\frac{g l_{p}+4}{g l_{p}}}\right)}{2 l_{p}}\right)}\right.}{2 l_{p}}-\frac{e^{\frac{t\left(-g l_{p}-g l_{p} \sqrt{\frac{g l_{p}+4}{g l_{p}}}\right)}{2 l_{p}}}+e^{\frac{t\left(g l_{p}-g l_{p} \sqrt{\frac{g l_{p}+4}{g l_{p}}}\right)}{2 l_{p}}}}{2 l_{p}}
\end{aligned}
$$

Taking the exact same steps for the system (8.2) we will get $u_{6}^{0}=u_{6}^{0}(t)$ which will be

$$
\begin{aligned}
u_{6}^{0} & =\frac{\left(e^{\left.\frac{t-g l-g l}{2 l} \sqrt{\frac{g l+4}{g l}}\right)}-e^{\left.\frac{t(g l-g l}{2 l} \sqrt{\frac{g l+4}{g l}}\right)}\right)}{l^{2}} \\
& +\frac{1}{4} \sqrt{\frac{g l+4}{g l}}\left(\sqrt{\frac{g l+4}{g l}} e^{\frac{t\left(-g l-g l \sqrt{\frac{g l+4}{g l}}\right.}{2 l}}-\sqrt{\frac{g l+4}{g l}} e^{\frac{t\left(g l-g l \sqrt{\frac{g l+4}{g l}}\right.}{2 l}}-e^{\frac{t\left(-g l-g l \sqrt{\frac{g l+4}{g l}}\right.}{2 l}}-e^{\frac{t\left(g l-g l \sqrt{\frac{g l+4}{g l}}\right.}{2 l}}\right)
\end{aligned}
$$

5. Back to Core Problem.

Now, that we have the solution for the subproblem, we can proceed to our main problem.

Recall that the control inputs in the sub problem which are $u_{5}^{0}=u_{5}^{0}(t)$ and $u_{6}^{0}=u_{6}^{0}(t)$ are 
actually $x_{7}$ and $x_{8}$ in the system (7). In that case one can notice that two subsystems of (7) can be simply integrated. Those subsystems are the following.

$$
\begin{aligned}
& \left\{\dot{x}_{1}=x_{2} ; \dot{x}_{2}=\frac{g}{2 l_{p}} x_{13} ; \dot{x}_{8}=x_{11} ; \dot{x}_{11}=\frac{u_{3}}{I_{y y}}-\frac{g}{I_{y y}} x_{13}\right. \\
& \left\{\dot{x}_{3}=x_{4} ; \dot{x}_{4}=\frac{g}{2 l_{p}} x_{15} ; \dot{x}_{7}=x_{10} ; \dot{x}_{10}=\frac{u_{2}}{I_{x x}}-\frac{g}{I_{x x}} x_{15}\right.
\end{aligned}
$$

As we already have $x_{7}=x_{7}(t)$ and $x_{8}=x_{8}(t)$ we can simply derive $x_{11}=x_{11}(t)$ from system (7.1) and $x_{10}=x_{10}(t)$ from system (7.2). As for $x_{i}=x_{i}(t), i=\overline{1,4}$, we will obtain by integrating $\dot{x}_{2}=\frac{g}{2 l_{p}} x_{13}$ and $\dot{x}_{4}=\frac{g}{2 l_{p}} x_{15}$ under the consideration of desired edge conditions. As a result, we will have the desired state trajectories of the UAV and the control inputs $u_{2}=u_{2}(t)$ and $u_{3}=u_{3}(t)$ which will drive the system through the desired trajectories. Of course, those control inputs are not optimal because of the absence of constraint.

The remaining two subsystems of (7) which are

$\left\{\dot{x}_{5}=x_{6} ; \dot{x}_{6}=u_{1}\right.$

$\left\{\dot{x}_{9}=x_{12} ; \dot{x}_{12}=\frac{u_{4}}{I_{z z}}\right.$

in this paper are not discussed. The reason is that these are independent of the dynamics of the pendulum in the linearized model of the system we have.

Another reason is that (7.3) and (7.4) are of no interest because of the simplicity of their solutions.

\section{Simulating the Results.}

We have chosen to check the theoretical result of this paper by simulating the motion of the UAV and recording state trajectories in form of graphs with time being the independent variable. For the simulation purposes the following values have been chosen for the parameters

$$
g=9.81 \mathrm{~m} \mathrm{~s}^{-2}, \quad l_{p}=1 \mathrm{~m}, \quad I_{x x}=I_{y y}=0.4856 \mathrm{Kg} \mathrm{m}^{2}
$$

Finally, we are ready to present the graphs describing the motion of the quadcopter (shown below).

In the Figures $2 \div 5$ the phase trajectories of quadrotor are shown, and in the Figure 10 and Figure 11 the phase trajectories of inverted pendulum are shown.

From the figures it follows that in this problem of controlling the movement of the system a quadrotor-inverted pendulum, the coordinates of the center of gravity of the quadrotor and the coordinates of the inverted pendulum increase (Figure 2 and Figure 4, Figure 10 and Figure 11) and the phase velocities of the center of gravity of the quadrotor are stabilized (Figure 3 and Figure 5).

In the Figure 6 and Figure 7 the control inputs $u_{2}(t)$ and $u_{3}(t)$ are given, that bring the system from a given initial position to a given final position. In the Figure 8 and Figure 9 the control inputs $u_{5}^{0}(t)$ and $u_{6}^{0}(t)$ are given, that bring the system from a given initial 
position to an asymptotically stable position while minimizing the linear quadratic controller.
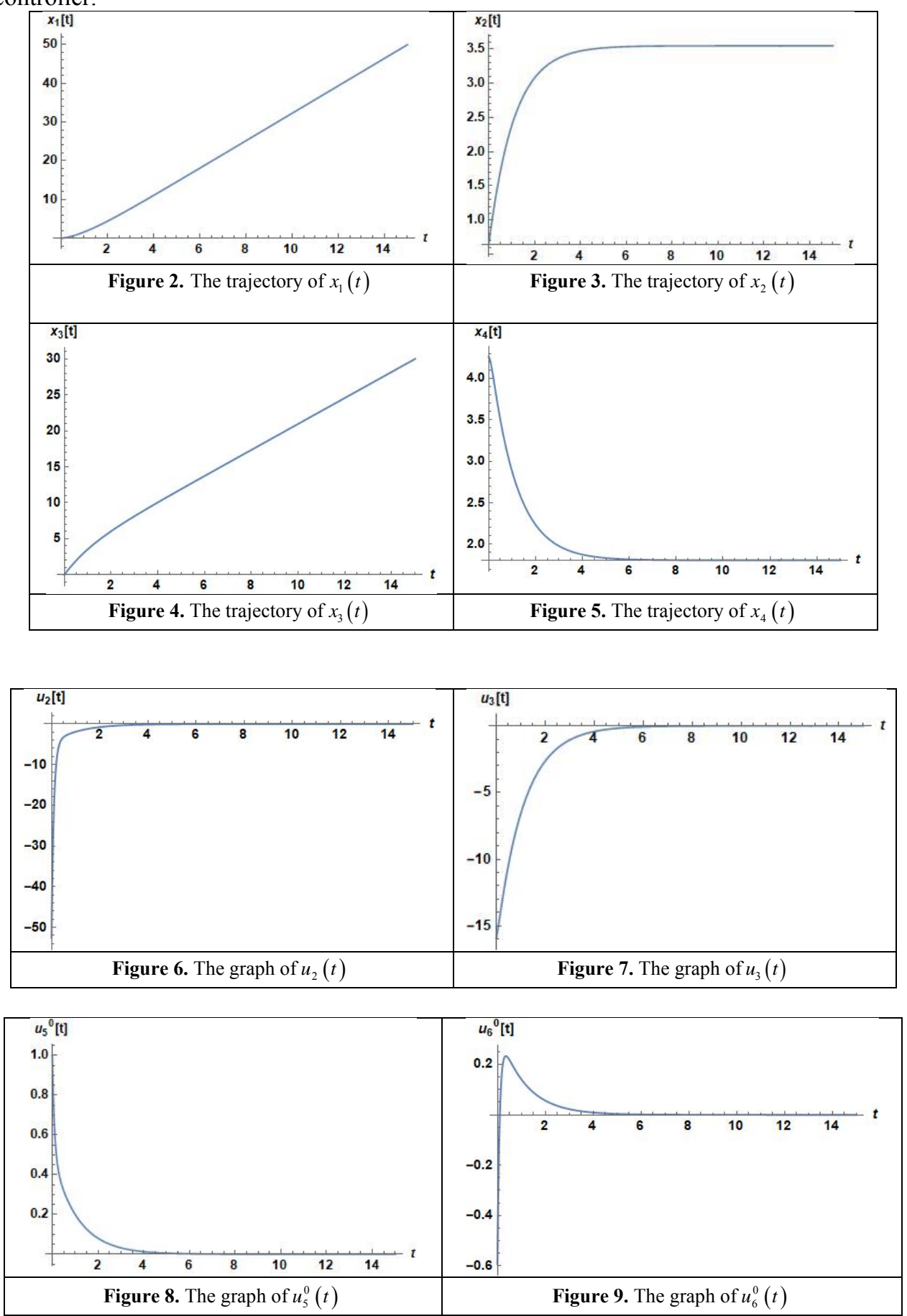


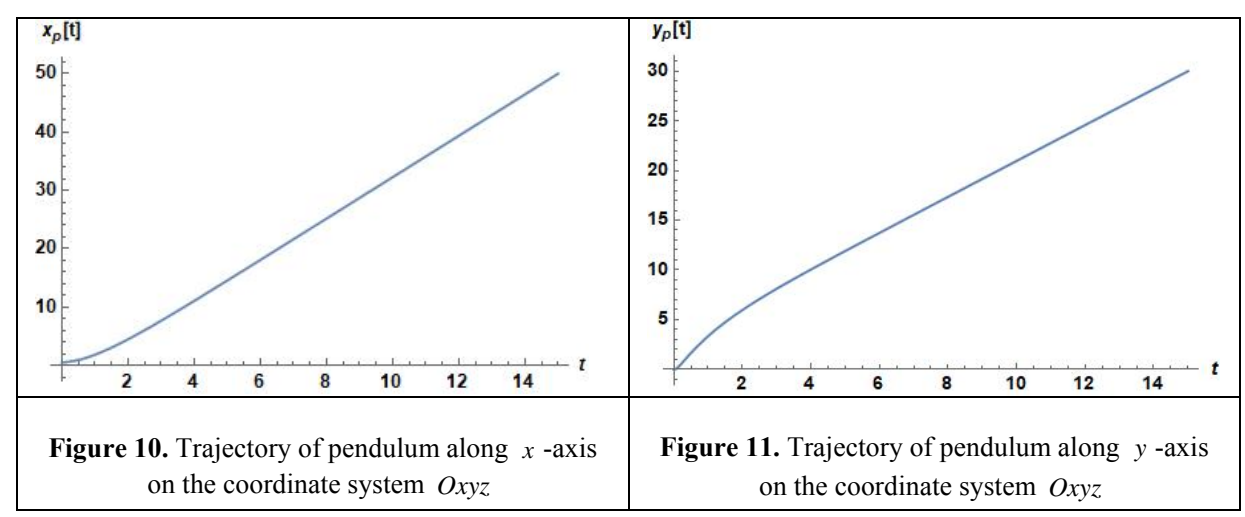

Obviously, the control inputs control inputs $u_{2}(t), u_{3}(t)$ and $u_{5}^{0}(t)$, quickly converge to zero. The control input $u_{6}^{0}(t)$ also quickly converges to zero, but after impulsive increase.

Figure 12 and Figure 13 show that how different the real trajectory of the UAV and optimal trajectory of a Pendulum-Free UAV in 3D space.

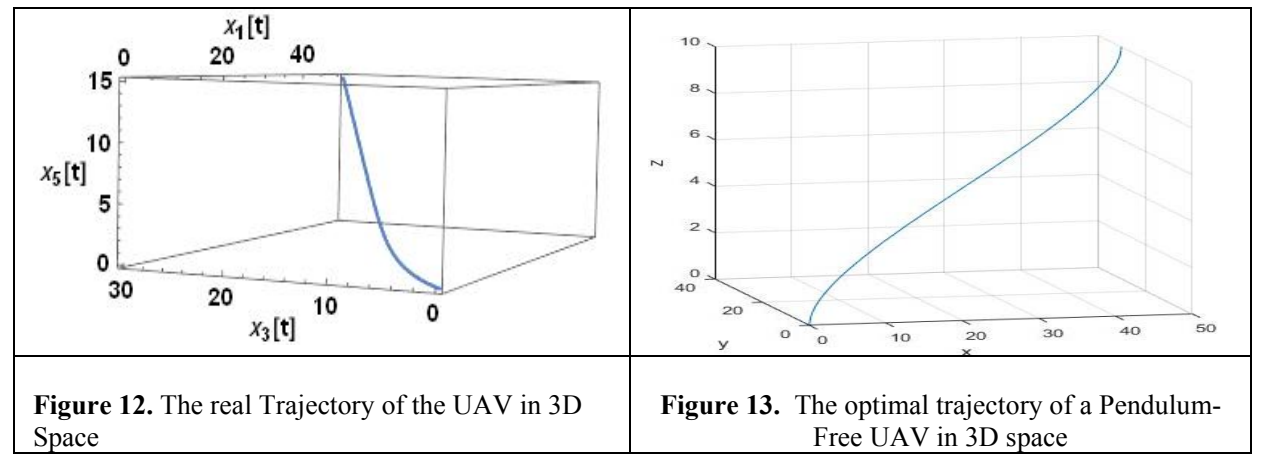

To compare this result with the case when there is no pendulum mounted on top of the UAV we will present the optimal trajectory of a free-of-pendulum UAV which is driven from the same initial point to the same final position.

\section{Conclusion}

The dynamics of the pendulum is presented with respect to the UAV and then both models are combined into one. The model is then linearized and the control problem is solved using proposed hybrid method, which means, we first stabilized optimally the pendulum using the motion of the UAV as control inputs and then we used the optimal stabilizing control inputs to drive the UAV-Pendulum system to a desired position. The results we gained are shown in form of graphs which were generated from virtual simulations.

\section{REFERENCES}

1. Ramiro Ibarra Pérez, Gerardo Romero Galvan, Aldo Jonathan Muñoz Vázquez, Silvia Florida Melo and David Lara Alabazares: Attitude Control of a Quadcopter Using Adaptive Control Technique: December 20th 2017 DOI: 10.5772/intechopen.71382.

2. Austin R. Unmanned Aircraft Systems: UAVS Design, Development and Deployment. John Wiley \& Sons. United Kingdom; 2010. p. 372 DOI: 10.1002/9780470664797 
3. Jeong S et al. Position Control of a Quad-Rotor System. In: Kim J-H, Matson ET, Myung $\mathrm{H}, \mathrm{Xu} \mathrm{P}$, editors. Robot Intelligence Technology and Applications. Berlin Heidelberg: Springer-Verlag; 2013; 2012. p. 971-981. DOI: doi.org/10.1007/978-3642-37374-9_94.

4. Bouabdallah $\overline{\mathrm{S}}$, editor. Design and control of quadrotor with application to autonomous flying [dissertation]. École Polytechnique Fédérale de Lausanne; 2007.

5. Jithu G, Jayasree PR. Quadrotor modelling and control. International Conference on Electrical, Electronics, and Optimization Techniques (ICEEOT) - 2016.

6. Jun L, Yuntang Li. Dynamic analysis and PID control for a quadrotor. In: Proceedings of the 2011 IEEE. 2011.

7. Changlong L, Jian P, Chang Y. PID and LQR trajectory tracking control for an unmanned quadrotor helicopter: Experimental studies. In: Proceedings of the 35 th Chinese Control Conference. 2016; DOI: 10.1109/ChiCC.2016.7555074.

8. Salazar S, Romero H, et al. Real-time stereo visual serving control of an UAV having eight rotors, In: 6th International Conference on Electrical engineering, computing science and automatic control, CCE 2009. DOI: 10.1109/ICEEE.2009.5393423.

9. Bouabdallah S, Siegwart R. Backstepping and sliding-mode techniques applied to an indoor micro quadrotor. In: Proceedings of the 2005 IEEE International Conference on Robotics and Automation, ICRA 2005. DOI: 10.1109/ROBOT.2005.1570447.

10. Abeywardena DMW, Amaratunga LAK, Shakoor SAA, Munasinghe SR. A velocity feedback fuzzy logic controller for stable hovering of a quadrotor UAV. In: International ConfDerence on Industrial and Information Systems (ICIIS), 2009. DOI: 10.1109/ICIINFS.2009.5429800.

11. Theerasak S, Pined L, Wonlop C, et al. Path tracking of UAV using self-tuning PID controller based on fuzzy logic, In: Proceedings of SICE Annual Conference 2010.

12. Hehn, Markus \& D'Andrea, Raffaello. (2011). A flying inverted pendulum. Proceedings - IEEE International Conference on Robotics and Automation. 763-770. 10.1109/ICRA.2011.5980244.

13. Luukkonen T., Modelling and Control of Quadcopter. School of Science, Mat-2.4108, Independent Research project in applied mathematics, Espoo, August 22, 2011, 26 p.

14. Shahinyan A.S., An Optimal Control Problem with Energy Constraint for an Unmanned Aerial Vehicle, IX international conference The Problems of Interaction of Deformable Media, Dedicated to the $75^{\text {th }}$ anniversary of NAS RA, October $1-6,2018$, Goris

15. Buchholz, N.N., The Main Course of Theoretical Mechanics, M.: the Science, h. 2, 1972, 332p. [in Russian].

\section{Сведение об авторе}

Шагинян Арман Смбатович - аспирант кафедры механики,

Ереванский государственный университет, факультет математики и механики,

(374 55) 66-37-41 E-mail armanshah1995@gmail.com

Received 11.03.2020 